\title{
Improving Prognostic and Fibrotic Evaluation of Chronic Kidney Disease with Contrast-Enhanced Ultrasound Index Derived Peak Intensity: an observational study
}

\section{Yao Xu}

Shanghai Jiao Tong University School of Medicine Affiliated Renji Hospital

Hongli Li

Shanghai Jiao Tong University School of Medicine Affiliated Renji Hospital

\section{Chunlin Wang}

Shanghai Jiao Tong University School of Medicine Affiliated Renji Hospital

\section{Minfang Zhang}

Shanghai Jiao Tong University School of Medicine Affiliated Renji Hospital

Qin Wang

Shanghai Jiao Tong University School of Medicine Affiliated Renji Hospital

\section{Yuanyuan Xie}

Shanghai Jiao Tong University School of Medicine Affiliated Renji Hospital

\section{Xinghua Shao}

Shanghai Jiao Tong University School of Medicine Affiliated Renji Hospital

\section{Lei Tian}

Shanghai Jiao Tong University School of Medicine Affiliated Renji Hospital

\section{Yanhong Yuan}

Shanghai Jiao Tong University School of Medicine Affiliated Renji Hospital

\section{Wei Yan}

Shanghai Jiao Tong University School of Medicine Affiliated Renji Hospital

\section{Tienan Feng}

Shanghai Jiao Tong University School of Medicine Affiliated Renji Hospital

\section{Fenghua Li}

Shanghai Jiao Tong University School of Medicine Affiliated Renji Hospital

\section{Zhaohui Ni}

Shanghai Jiao Tong University School of Medicine Affiliated Renji Hospital

Shan Mou ( $\nabla$ shan_mou@shsmu.edu.cn )

Shanghai Jiao Tong University School of Medicine Affiliated Renji Hospital https://orcid.org/00000003-4160-1681 


\section{Research article}

Keywords: contrast-enhanced ultrasound CEUS; chronic kidney disease CKD; derived peak intensity DPI; renal biopsy; sulphur hexafluoride microbubbles contrast SonoVue

Posted Date: August 23rd, 2019

DOl: https://doi.org/10.21203/rs.2.13521/v1

License: (c) (i) This work is licensed under a Creative Commons Attribution 4.0 International License.

Read Full License 


\section{Abstract}

Background Chronic kidney disease (CKD) is a progressive disease with high morbidity and mortality. Contrast-enhanced ultrasonography (CEUS) has emerged as a cost-effective novel non-invasive imaging technique to improve the diagnosis and predict CKD progression. Methods Patients diagnosed with CKD who underwent CEUS with intravenous bolus contrast $1.5 \mathrm{ml}$ SonoVue during 2014 to December 2014 at Ren Ji hospital, Shanghai, China, were enrolled in this study. Time-intensity curves and quantitative indexes were created using QLAB quantification software. Kidney biopsies were analyzed with a-SMA immunohistochemistry. The Cox proportional hazards model was used to investigate the risk factors for kidney survival. Results Out of 167 CKD patients followed for a median of $23.6 \pm 11.3$ months, 29 (17.4\%) exhibited CKD progression with decline in glomerular filtration rate of more than $25 \%$ or end-stage renal disease. Multivariate Cox regression analysis revealed lower derived peak intensity (DPI) was independently associated with progression of kidney disease. Patients with $\mathrm{DPI} \leq 11.98 \mathrm{db}$ had more severe renal fibrosis and less likely to recover from CKD progression. Conclusions This study demonstrated that the DPI is a reliable CEUS parameter for the evaluation of renal function and independent predictor for the prognosis of CKD patients.

\section{Introduction}

Chronic kidney disease (CKD) is a progressive disease and an important public health challenge with high morbidity and mortality associated with end-stage renal disease (ESRD), cardiovascular disease, and premature death leading to increased health care cost and decreased quality of life [1-4]. The 2013 Global Burden of Disease ranked CKD as 19th causes of deaths (age-standardized annual death rate of 15.8 per 100,000 individuals), a substantial increase from 36 th in $1990(11.6$ per 100,000) [5].

The reduction in microvascular blood flow, focal hypoxia and inflammation leads to fibrogenesis and progression to CKD [6]. Non-invasive imagings, such as contrast-enhanced computed tomography (CECT) and magnetic resonance imaging (MRI) used to evaluate renal perfusion have limitation of patient cooperation, equipment availability, costs and risks of contrast-induced nephropathy. Grey-scale renal ultrasound combined with color Doppler flow imaging (CDFI) is non-invasive evaluation of renal perfusion [7]. But, CDFI is influenced by manipulations, patient coordination, obesity and only provides the information on global organ perfusion, not the microcirculation which is important in CKD progression $[8,9]$.

The contrast-enhanced ultrasonography (CEUS) is increasingly used to evaluate microcirculation and perfusion in different organs [10]. The SonoVue, a sulphur hexafluoride microbubbles blood-pool CEUS contrast is stable in the circulatory system. With a short half-life of a few minutes and excreted via lungs, is suitable to evaluate renal microvascular blood flow [11]. This is safe, non-invasive, convenient, and can be used repeatedly [10]. 
The purpose of this study was to analyze the ability of CEUS to detect and predict renal function changes in CKD patients.

\section{Materials And Methods}

\section{Study Population}

This was a prospective cohort study of CKD patients admitted in the nephrology department of Ren Ji Hospital, Jiao Tong University, Shanghai, China, between January 2014 and December 2014. The CKD was defined as abnormality of the kidney structure or function that had persisted for at least three months with effects on patient health according to the Kidney Disease Improving Global Outcomes (KDIGO) clinical practice guidelines for CKD [12]. Written informed consent was obtained. The study was conducted in accordance with the Declaration of Helsinki, and approved by the Ethics Committee of Ren Ji Hospital.

\section{Enrollment Criteria}

The inclusion criteria were (1) CKD patients admitted in hospital; (2) age 18 to 80 years; (3) had CEUS and renal biopsy; (4) follow-up of at least six months after biopsy; (5) body mass index (BMI) between 18.5 to 27.9. The exclusion criteria were patients with: (1) incomplete data; (2) contrast agent allergy; (3) urolithiasis or renal tumor; (4) pneumonia or pleural effusion; (5) asthma, chronic bronchitis, chronic obstructive pulmonary disease, or cardiopulmonary dysfunction; (6) pregnancy; (7) poor breath-hold or uncooperative.

\section{Ultrasound Examination}

The conventional ultrasound and CEUS was performed with 1-5 MHz transducer on Philips iU22 ultrasound (Philips, Bothell, WA, USA) by a single operator with expertise and more than 10 years of work experience. The operator was blinded to the renal function of patients.

To standardize the study protocol, we chose to perform ultrasound of the left kidney (for the convenience of right handed operator, and only on single kidney to reduce the time for the investigation) on all patients enrolled in the study. Grey-scale ultrasound was used to evaluate and record the size, shape, echogenicity and thickness of the renal cortex. The renal cortex thickness was measured perpendicular to the renal cortex, from the base of medullary pyramid to the inner margin of the renal capsule. Acoustic Radiation Force Impulse Imaging (ARFI) was used to detect the elasticity index (EI) and assess the elastic stiffness of renal tissue. Each patient's kidney was measured three times in the upper, middle and lower poles and the mean value was calculated. The renal segment artery resistance index (RI) was measured by CDFI. After a conventional ultrasound examination, the contrast agent SonoVue (BR1; Bracco Milan, Italy) bolus of $1.5 \mathrm{ml}$ was injected followed by $5 \mathrm{ml}$ of normal saline via peripheral intravenous cannula, and the renal perfusion images were recorded continuously within 3 minutes. 
The region of interest (ROI) images were analyzed with QLAB quantification software (Philips) and Timeintensity curves (TICs) created with Gamma Variate fitting. The same area and size $(5 \mathrm{~cm} \times 5 \mathrm{~cm})$ of ROI sampling frame was placed in the renal cortex perpendicular to wave, and the same depth and position were maintained as far as possible among different patients. TIC was generated automatically to evaluate the relevant quantitative indexes, including area under curve (AUC) of TIC (positively correlation with renal blood flow), time to peak (TTP, the time to peak renal enhancement), derived peak intensity (DPI, the peak renal enhancement value), time from peak to one half and arrival time (AT, the time required for the contrast agent to arrive at the renal cortex).

\section{Histological Examination}

The biopsy was performed in the left kidney under ultrasound guidance within 3 days of the ultrasound examination. All the biopsy specimens were processed the same centre (Ren Ji Hospital, Shanghai, China) and reported by a single pathologist with expertise and experience of 10 years. The pathologist was blinded to ultrasound and CEUS findings. Epinephrine and antihypertensive drugs were kept handy during the biopsy procedure to manage untoward accidents.

The biopsies were fixed with $4 \%$ formaldehyde and embedded in paraffin. For immunohistochemical staining, the paraffin sections were cut into $3 \mathrm{~mm}$ and boiled in $10 \mathrm{mM}$ Na citrate solution (pH6.0) for 10 minutes and stained with a smooth muscle actin antibody (-SMA; 1:400; Abcam, Cambridge, Massachusetts). All non-overlapping fields of images were captured $(\times 100)$ and analyzed using ImagePro Plus (version 6.0; Media Cybernetics, Silver Spring, MD, USA). The degree of renal interstitial fibrosis was evaluated as the density mean of -SMA (integrated option density (IOD)/area of interest (AOI)) [13].

\section{Data Collection and Patient Follow-Up}

Patients underwent regular follow-up visits after the renal biopsy every three months by two nephrologists in the clinic of Ren Ji Hospital, Shanghai, China, until December 2017. Baseline data were recorded at the time of admission. Demographic data included age, sex and BMI. Clinical and laboratory parameters were obtained at baseline and during the follow-up, such as follow-up duration, time to endpoint, mean arterial blood pressure (MAP), 24-hour urinary protein excretion (UPE), HbA1c, serum creatinine (SCr). Proteinuria was detected by 24 -hour urine protein collection. The estimated glomerular filtration rate (eGFR) was estimated according to the Chronic Kidney Disease Epidemiology Collaboration equation [14, 15].

The start of follow-up period was the date of kidney biopsy. The composite kidney failure events were defined as CKD progression based on a $25 \%$ or greater drop in eGFR without remission or ESRD with eGFR $<15 \mathrm{ml} / \mathrm{min} / 1.73 \mathrm{~m} 2$, dialysis, or kidney transplant [12].

\section{Statistical Analyses}

The SPSS 22.0 (IBM, USA) was used for data analysis. Continuous variables were expressed as means $\pm S D$ and median $(M)$ and interquartile range (IQR, P25, P75). Categorical variables were expressed as frequencies or percentages. Relevant risk factors that might affect renal prognosis were analyzed via 
Cox analysis [16-20]. The multivariate models were created through a stepwise backward selection procedure based on a likelihood ratio, with $\mathrm{P}<0.05$ for the inclusion of the variables of CEUS. The sensitivity and specificity of variables for renal prognosis were evaluated using receiver operating characteristic (ROC) curves. The Kaplan-Meier method was used to estimate kidney survival, and comparisons were performed by the log-rank test. Spearman correlation analysis was used to analyze correlations. A value of $\mathrm{P}<0.05$ was considered statistically significant.

\section{Results}

\section{Clinical Characteristics}

Between January 2014 and December 2014, 245 eligible patients were consecutively enrolled, among which 78 were excluded; because of unilateral renal atrophy (4), co-existing urolithiasis or renal tumor (11) or lost to follow-up (63). Finally, data on 167 patients (87 males, 80 females) with CEUS and renal biopsies were analyzed, (Figure 1). Postoperative complications such as lumbago or microscopic hematuria seen in some patients resolved spontaneously.

Twenty-nine patients showed progression of CKD at during the end of the follow-up $23.6 \pm 14.1$ months (minimum 6 months) during the 4 years study period. The clinical characteristics at baseline showed mean age of CKD patients at first kidney biopsy was $43.6 \pm 14.8$ years, BMI $24.8 \pm 3.7 \mathrm{~kg} / \mathrm{m} 2$, MAP was $96.3 \pm 11.3 \mathrm{mmHg}$, median and IQR of initial proteinuria1.7 and $0.8-4.0 \mathrm{~g} / \mathrm{d}$, baseline HbA1c $5.5 \pm 1.0 \%$, SCr and eGFR $104.4 \pm 91.1 \mu \mathrm{mol} / \mathrm{l}$ and $83.7 \pm 30.6 \mathrm{ml} / \mathrm{min}$ per $1.73 \mathrm{~m} 2$, respectively, (Table 1 ).

\section{Ultrasound Parameters}

The conventional and color Doppler ultrasound of left kidneys revealed, mean renal length and width of $107.0 \pm 8.4$ and $48.9 \pm 6.38 \mathrm{~mm}$ and cortical thickness of $9.29 \pm 2.03 \mathrm{~mm}$, (Table 1). The mean RI was $0.62 \pm 0.05 \mathrm{~g} / \mathrm{dL}, A R F I$ evaluation of renal elasticity median peak El $2.5 \mathrm{~m} / \mathrm{s}(\mathrm{IQR}=2.1-2.9)$, mean AT of SonoVue to arrive at the renal cortex and from peak to one half were $13.8 \pm 4.2$ and $64.1 \pm 26.3 \mathrm{~s}$, respectively and mean DPI $12.9 \pm 3.5 \mathrm{db}$, (Table 1$)$.

\section{Kidney Progression and Risk Factors}

By the last visit, maximum of 3-years, out of 167 CKD patients 29 (17.4\%) composite kidney failure events were observed. The clinical features and CEUS parameters associated with the risk of CKD progression according to the univariate analyses were advancing age, higher $\mathrm{HbA} 1 \mathrm{c}$, declining renal function and more severe proteinuria, (Table 2). Ultrasound parameters of renal atrophy, decreased cortical thickness, longer TTP and lower DPI were risk factors for progression of CKD and poor kidney function; whereas other parameters, such as RI and AUC did not significantly correlate with the prognosis. After adjusting for the age, BMI, eGFR, MAP, HbA1c and UPE; the multivariate analysis showed lower DPI had significantly increased risk of CKD progression (HR 0.86, 95\% $\mathrm{Cl} 0.74,0.99, \mathrm{P}=0.03$ ).

\section{The ROC curve analysis}


At the cut off value of $11.98 \mathrm{db}$, the area under the ROC of (1) DPI was $0.65(95 \% \mathrm{Cl}, 0.57-0.72, \mathrm{P}=0.01)$ with a sensitivity $65.5 \%$ and specificity of $61.5 \%$; (2) UPE was 0.69 (95\%Cl, 0.61-0.76, P=0.02); (3) HbA1c was $0.65(95 \% \mathrm{Cl}, 0.57-0.72, \mathrm{P}=0.04)$. When $\mathrm{DPI}$, UPE and $\mathrm{HbA} 1 \mathrm{c}$ were substituted in the multiple factor logistic regression model, the area under the ROC of the combined parameter was $0.80(95 \% \mathrm{Cl}, 0.73-0.86$, $\mathrm{P}<0.01$ ), with a sensitivity of $77.8 \%$ and a specificity of $75.4 \%$, which had the largest AUC, (Figure 2 , Table 3).

\section{Relationship between DPI and renal prognosis}

At $11.98 \mathrm{db}$ as the cut-off value, patients with $\mathrm{DPI} \leq 11.98 \mathrm{db}$ were positively associated with a significantly poor kidney outcome ( $p<0.01$ ) (Figure 3), compared to DPI >11.98db (Table 4). The renal biopsy of patients with $\mathrm{DPI} \leq 11.98 \mathrm{db}$ had more intense -SMA positive staining, indicating sever renal fibrosis. (Figure 4)

\section{Relationship between DPI and renal fibrosis}

The spearman correlation analysis demonstrated that DPI had a modest but statistically significant correlation with the degree of renal fibrosis according to -SMA expression ( $r=-0.37, p<0.01)$, (Figure 5). Patients with a lower DPI exhibited increased renal fibrosis. Patients with median -SMA positive staining of $1.63 \%$ area of interstitium as cutoff value, the AUC of DPI to assess renal fibrosis was $0.67(95 \% \mathrm{Cl}$, $0.58-0.75, P<0.01$ ) with a sensitivity of $67 \%$ and a specificity of $61 \%$, (Figure 6 ).

\section{Discussion}

With application of novel non-invasive SonoVue contrast imaging technique of CEUS, we found that CKD patients with low DPI $(\leq 11.98 \mathrm{db})$ were more likely to have poor renal outcome. The Cox regression analysis revealed a low DPI as an independent risk of nephropathy progression after adjusting for clinical features. With an AUC of 0.80 , the DPI combined with UPE and HbA1c could accurately predict renal function prognosis. The Kaplan-Meier curves revealed that DPI had a satisfactory ability to estimate the risk of kidney progression for CKD patients and thus, the DPI can be used as a marker to predict prognosis of CKD. Recent researches have demonstrated CEUS is a novel imaging to assess renal perfusion and Jin et al. reports CEUS parameters were ideal markers for monitoring the perfusion status of transplanted kidneys in 57 renal transplant patients, for acute rejection (AR) and non-AR [21]. Dong et al. have suggested that the DPI might be a reliable quantitative index and can be promoted for the early diagnosis of diabetic nephropathy (DN) [11]. However, there is lack of published reports and the role of CEUS in CKD is not clear in literature.

The DPI levels in our study had high correlation with the degree of renal fibrosis $(\mathrm{P}<0.01)$. Lower DPI values indicated more severe renal fibrosis. We stained the tissue sections with -SMA to evaluate the degree of renal fibrosis, pathological damages and corresponding DPI levels. The ROC curve indicated that the DPI can be used as a marker of renal fibrosis with an AUC of 0.67. Thus, DPI may be a valuable alternative to the renal biopsy, which is currently the gold standard for CKD diagnosis. The histological findings of renal fibrosis, characterized by glomerular sclerosis and tubulointerstitial fibrosis, is the 
common pathological consequence of a wide variety of CKDs [22, 23]. Biopsy is invasive procedure with risks of complications, contraindicated in some patients, patients and family are often reluctant for the invasive tests and it has limitations for repeated use and reproducibility.

Our results demonstrate the usefulness of the DPI as a strong predictor of progress of CKD. When renal fibrosis occurs, microcirculation perfusion is decreased. Thus, a reduced amount of microbubbles enters the renal cortex, resulting in a reduced echo decibel from the renal cortex, which results in a decreased DPI. The DPI, defined as the maximum echo decibel of the TIC, assesses the perfusion of organs. In animal model, Ying $\mathrm{M}$ et al. showed that the measurement of the liver parenchymal DPI was helpful for the evaluation of liver fibrosis, and the DPI decreased with advancing stages of fibrosis [24]. Atri $\mathrm{M}$ et al. found that the DPI increased significantly in malignant renal masses compared to benign with visible changes in vascularity [25].

We used SonoVue contrast agent in current study. This agent consists of an inert gas embedded within a shell (phospholipids or albumin), which forms microbubbles of 1 to $6 \mu \mathrm{m}$ size after intravenous injection. The size of the bubbles prevents them from diffusing through the endothelium, and are highly stable in the blood. Behaving as blood-pool agents, they undergo the same intravascular rheology as red blood cells. As a result, microbubbles reflect microcirculation perfusion $[10,26]$. Currently, CEUS has been accepted as a new imaging technique for quantifying microcirculation perfusion in the liver, kidney, and other organs [27-29].

In the kidney, the animal study conducted by Schlosser et al. showed that the acquisition of replenishment curves using CEUS was helpful for differentiating macro- and microcirculation [30]. The renal microvasculature is a crucial component in renal fibrosis and CKD progression. Unlike renal tubules, the microvasculature lacks the capacity for regeneration, which results in persistent peritubular capillary regression after severe or recurrent injury. Loss of tissue perfusion due to glomerular sclerosis, inflammatory infiltration, and the toxicity of filtered proteins contribute to the loss of renal microvasculature. The subsequent reduction of microvascular blood flow induces tissue focal hypoxia and ultimately causes renal fibrosis [6, 31]. Based on our findings of SonoVue CEUS index DPI in CKD patients, we propose that CEUS can be used as a fast, sensitive, and reliable non-invasive technique to assess renal fibrosis and predict renal progression.

Some of the limitations of our study may be a single-center tertiary care teaching hospital site in urban Shanghai, and may not be generalized. However, this single center study ensures the consistency of diagnostic criteria of CEUS and reduces the possible discrepancies caused by different operators and instruments compared to multicenter studies. Second, we did not include the information on the influence of different treatments received by patients. Out of total 245 CKD patients who had CEUS, 63 (25.7\%) were excluded from the final analysis due to incomplete follow-up data (28), no follow-up (12) and less than 6 months follow up (23), which could be improved in future by increasing sample size and having dedicated persons to call the patients by phone for follow-up to minimize data loss.

\section{Conclusions}


In conclusion, our study demonstrates that DPI is a reliable CEUS parameter for the evaluation of renal function and is independent predictor for the prognosis of CKD patients. The low DPI indicates a high possibility of poor renal outcome. This method combines the advantages of being safe, non-invasive, convenient, and suitable for repeated use in routine clinical practice. The DPI can be used as a rapid and continuous indicator of renal dysfunction in CKD patients.

\section{List Of Abbreviations}

CEUS, contrast-enhanced ultrasound; CKD, chronic kidney disease; ESRD, end-stage renal disease; CT, computed tomography; MRI, magnetic resonance imaging; CDFI, color Doppler flow imaging; GFR: glomerular filtration rate; BMI, body mass index; MAP, mean arterial blood pressure; ALB, serum albumin; UPE, 24-h urinary protein excretion; ACR, albumin creatinine ratio; SCr, serum creatinine; UA, plasma urea; BUN, blood urea nitrogen; RI, resistance index; AT, arrival time; TTP, time to peak; DPI, derived peak intensity; AUC, area under curve; $\mathrm{PI}$, prognostic index.

\section{Declarations}

\section{Ethics approval and consent to participate}

The study was conducted in accordance with the Declaration of Helsinki, and approved by the Ethics Committee of Ren Ji Hospital.

\section{Consent for publication}

Not applicable

\section{Availability of data and materials}

The datasets used and/or analysed during the current study are available from the corresponding author on reasonable request.

\section{Competing interests}

The authors have no conflicts of interest to declare.

\section{Funding}

Authors must give full details about the funding of any research relevant to their study, including sponsor names and explanations of the roles of these sources in the preparation of data or the manuscript. This study was supported in part by Science \& Technology Cooperation Program of China (2017YFE0110500). The study was also sponsored by the National Natural Science Foundation of China (81373865, $81573748 \varangle 81770668)$ as well as by a grant (14140903200) from the Science and Technology Commission of Shanghai Municipality, China. The study was also sponsored by the Program of 
Shanghai Academic Research Leader (16XD1401900) and a grant ([2017]485) from the Shanghai Leadership Training Program.

\section{Acknowledgements}

We thank the doctors at the Nephrology and Ultrasound Department of Ren Ji Hospital for their work and support to complete this study. We acknowledge the review of final manuscript and suggestion provided by Dr. Jay Shah, the visiting prof at Ren Ji hospital.

\section{References}

1. Mills KT, Xu Y, Zhang W, Bundy JD, Chen CS, Kelly TN, Chen J, He J: A systematic analysis of worldwide population-based data on the global burden of chronic kidney disease in 2010. Kidney Int 2015,88(5): 950-957.

2. Garcia-Garcia G, Jha V; World Kidney Day Steering Committee: CKD in disadvantaged populations. Can J Kidney Health Dis 2015,2: 18.

3. Yang W, Xie D, Anderson AH, Joffe MM, Greene T, Teal V, Hsu CY, Fink JC, He J, Lash JP, Ojo A, Rahman M, Nessel L, Kusek JW, Feldman HI; CRIC Study Investigators: Association of Kidney Disease Outcomes With Risk Factors for CKD: Findings From the Chronic Renal Insufficiency Cohort (CRIC) Study. Am J Kidney Dis 2014,63(2): 236-243.

4. Zhang L, Wang F, Wang L, Wang W, Liu B, Liu J, Chen M, He Q, Liao Y, Yu X, Chen N, Zhang JE, Hu Z, Liu F, Hong D, Ma L, Liu H, Zhou X, Chen J, Pan L, Chen W, Wang W, Li X, Wang H: Prevalence of chronic kidney disease in China: a cross-sectional survey. Lancet 2012,379(9818): 815-822.

5. GBD 2013 Mortality and Causes of Death Collaborators: Global, regional, and national age-sex specific all-cause and cause-specific mortality for 240 causes of death, 1990-2013: a systematic analysis for the Global Burden of Disease Study 2013. 2015,Lancet 385(9963): 117-171.

6. Ballermann BJ, Obeidat M: Tipping the balance from angiogenesis to fibrosis in CKD. Kidney Int Suppl (2011) 2014,4(1): 45-52.

7. Kavakli HS, Koktener A, Yilmaz A: Diagnostic value of renal resistive index for the assessment of renal colic. Singapore Med J 2011,52(4): 271-273.

8. Schneider AG, Hofmann L, Wuerzner G, Glatz N, Maillard M, Meuwly JY, Eggimann P, Burnier M, Vogt B: Renal perfusion evaluation with contrast-enhanced ultrasonography. Nephrol Dial Transplant 2012,27(2):674-681.

9. Dong Y, Wang WP, Cao J, Fan P, Lin X. Early assessment of chronic kidney dysfunction using contrastenhanced ultrasound: a pilot study. Br J Radiol 2014,87(1042): 20140350. 
10. Schneider A, Johnson L, Goodwin M, Schelleman A, Bellomo R: Bench-to-bedside review: contrast enhanced ultrasonography--a promising technique to assess renal perfusion in the ICU. Crit Care 2011,15(3):157.

11. Dong $Y$, Wang WP, Lin P, Fan P, Mao F: Assessment of renal perfusion with contrast-enhanced ultrasound: Preliminary results in early diabetic nephropathies. Clin Hemorheol Microcirc 2015,62(3): 229238.

12. KDIGO 2012 Clinical Practice Guideline for the Evaluation and Management of Chronic Kidney Disease. Kidney Int Suppl (2011) 2013,3(1): 19-63.

13. Ko GJ, Boo CS, Jo SK, Cho WY, Kim HK: Macrophages contribute to the development of renal fibrosis following ischaemia/reperfusion-induced acute kidney injury. Nephrol Dial Transplant 2008,23(3):842-52. 14. Levey AS, Stevens LA, Schmid CH, Zhang YL, Castro AF 3rd, Feldman HI, Kusek JW, Eggers P, Van Lente F, Greene T, Coresh J; CKD-EPI (Chronic Kidney Disease Epidemiology Collaboration): A New Equation to Estimate Glomerular Filtration Rate. Ann Intern Med 2009,150(9): 604-612.

15. Li X, Liu Y, Lv J, Shi S, Liu L, Chen Y, Zhang H: Progression of IgA nephropathy under current therapy regimen in a Chinese population. Clin J Am Soc Nephrol 2014,9(3): 484-489.

16. Halbesma N, Jansen DF, Heymans MW, Stolk RP, de Jong PE, Gansevoort RT, PREVEND Study Group: Development and validation of a general population renal risk score. Clin J Am Soc Nephrol 2011,6(7): 1731-1738.

17. Skupien J, Warram JH, Smiles A, Galecki A, Stanton RC, Krolewski AS: Improved glycemic control and risk of ESRD in patients with type 1 diabetes and proteinuria. J Am Soc Nephrol 2014,25(12): 2916-2925.

18. Grams ME, Sang Y, Ballew SH, Gansevoort RT, Kimm H, Kovesdy CP, Naimark D, Oien C, Smith DH, Coresh J, Sarnak MJ, Stengel B, Tonelli M; CKD Prognosis Consortium: A Meta-analysis of the Association of Estimated GFR, Albuminuria, Age, Race, and Sex With Acute Kidney Injury. Am J Kidney Dis 2015,66(4): 591-601.

19. Sandsmark DK, Messé SR, Zhang X, Roy J, Nessel L, Lee Hamm L, He J, Horwitz EJ, Jaar BG, Kallem RR, Kusek JW, Mohler ER 3rd, Porter A, Seliger SL, Sozio SM, Townsend RR, Feldman HI, Kasner SE; CRIC Study Investigators: Proteinuria, but Not eGFR, Predicts Stroke Risk in Chronic Kidney Disease: Chronic Renal Insufficiency Cohort Study. Stroke 2015,46(8): 2075-2080.

20. Yuan Y, Wang C, Shao X, Wang Q, Che X, Zhang M, Xie Y, Tian L, Ni Z, Mou S: Urinary retinol-binding protein as a risk factor of poor prognosis in acute on chronic renal injury. J Nephrol 2016,29(6):827-833.

21. Jin Y, Yang C, Wu S, Zhou S, Ji Z, Zhu T, He W: A novel simple noninvasive index to predict renal transplant acute rejection by contrast-enhanced ultrasonography. Transplantation 2015,99(3): 636-41. 
22. Liu Y: Renal fibrosis: new insights into the pathogenesis and therapeutics. Kidney Int 2006,69(2): 213217.

23. Chawla LS, Kimmel PL: Acute kidney injury and chronic kidney disease: an integrated clinical syndrome. Kidney Int 2012,82(5): 516-524.

24. Ying M, Leung G, Lau TY, Tipoe GL, Lee ES, Yuen QW, Huang YP, Zheng YP: Evaluation of liver fibrosis by investigation of hepatic parenchymal perfusion using contrast-enhanced ultrasound: an animal study. J Clin Ultrasound 2012,40(8): 462-470.

25. Guibal A TL, Mulé S, Comperat E, Comperat E, Badachi Y, Golmard JL, Le Guillou-Buffello D, Rixe O, Bridal SL, Lucidarme O: Noninvasive contrast-enhanced US quantitative assessment of tumor microcirculation in a murine model: effect of discontinuing anti-VEGF therapy. Radiology 2010,254(2): 420-429.

26. Granata A, Zanoli L, Insalaco M, Valentino M, Pavlica P, Di Nicolò PP, Scuderi M, Fiorini F, Fatuzzo P, Bertolotto M: Contrast-enhanced ultrasound (CEUS) in nephrology: Has the time come for its widespread use? Clin Exp Nephrol 2015,19(4): 606-615.

27. Ridolfi F, Abbattista T, Busilacchi P, Brunelli E: Contrast-enhanced ultrasound evaluation of hepatic microvascular changes in liver diseases. World J Gastroenterol 2012,18(37): 5225-5230.

28. Ma F, Cang Y, Zhao B, Liu Y, Wang C, Liu B, Wu T, Song Y, Peng A: Contrast-enhanced ultrasound with SonoVue could accurately assess the renal microvascular perfusion in diabetic kidney damage. Nephrol Dial Transplant 2012,27(7): 2891-2898.

29. Chen M, He Y, Zhang P, Geng Q, Liu Q, Kong L, Chen Y, Wei Q, Liu J, Guo S, Liu H: Comparison of Uterine Receptivity between Fertile and Unexplained Infertile Women by Assessment of Endometrial and Subendometrial Perfusion Using Contrast-Enhanced Ultrasound: Which Index is Better-Peak Intensity or Area under the Curve? Ultrasound in Medicine \& Biology 2016,42(3): 654-663.

30. Schlosser T, Pohl C, Veltmann C, Lohmaier S, Goenechea J, Ehlgen A, Köster J, Bimmel D, KuntzHehner S, Becher $\mathrm{H}$, Tiemann K: Feasibility of the flash-replenishment concept in renal tissue: which parameters affect the assessment of the contrast replenishment? Ultrasound Med Biol 2001,27(7): 937944.

31. Kramann R, Tanaka M, Humphreys BD. Fluorescence microangiography for quantitative assessment of peritubular capillary changes after AKI in mice. J Am Soc Nephrol 2014,25(9): 1924-1931.

\section{Tables}

Table 1. Baseline characteristics of chronic kidney disease (CKD) patients $(n=167)$ who had contrast-enhanced ultrasonography (CEUS) to detect and predict renal function changes 


\begin{tabular}{|c|c|}
\hline Characteristics & Value \\
\hline Age (years), mean \pm SD (median, IQR) & $43.6 \pm 14.8(42,30-56)$ \\
\hline Men, n (\%) & $87(52.1)$ \\
\hline $\mathrm{BMI}(\mathrm{kg} / \mathrm{m} 2)$, mean $\pm \mathrm{SD}$ (median, IQR) & $24.8 \pm 3.7(24.5,22.5-26.5)$ \\
\hline Baseline MAP (mmHg), mean \pm SD (median, IQR) & $96.3 \pm 11.3(94.7,88.7-103.3)$ \\
\hline HbA1c (\%), mean \pm SD (median, IQR) & $5.5 \pm 1.0(5.3,5.1-5.6)$ \\
\hline UPE (g/L), mean \pm SD (median, IQR) & $3.0 \pm 3.1(1.7,0.8-4.0)$ \\
\hline $\operatorname{Scr}(\mu \mathrm{mol} / \mathrm{l})$, mean $\pm \mathrm{SD}($ median, IQR) & $104.4 \pm 91.1(81.1,65.3-108.6)$ \\
\hline eGFR (ml/min per $1.73 \mathrm{~m} 2)$, mean \pm SD (median, IQR) & $83.7 \pm 30.6(89.9,61.4-107.0)$ \\
\hline$\alpha-S M A(\%)$, mean \pm SD (median, IQR) & $1.84 \pm 1.14(1.63,1.00-2.46)$ \\
\hline \multicolumn{2}{|l|}{ Ultrasound parameters, mean \pm SD (median, IQR) } \\
\hline Length $(\mathrm{mm})$, mean $\pm \mathrm{SD}$ (median, IQR) & $107.0 \pm 8.4(106.0,102.0-112.0)$ \\
\hline Width $(\mathrm{mm})$, mean \pm SD (median, IQR) & $48.9 \pm 6.4(49.0,45.0-53.0)$ \\
\hline Cortical thickness (mm), mean \pm SD (median, IQR) & $9.29 \pm 2.03(9.30,8.0-10.0)$ \\
\hline $\mathrm{RI}(\mathrm{g} / \mathrm{dL})$, mean $\pm \mathrm{SD}$ (median, IQR) & $0.62 \pm 0.05(0.62,0.60-0.65)$ \\
\hline $\mathrm{AT}(\mathrm{s})$, mean $\pm \mathrm{SD}$ (median, IQR) & $13.8 \pm 4.2(13.0,11.0-16.0)$ \\
\hline TTP (s), mean \pm SD (median, IQR) & $26.2 \pm 6.8(25.0,21.0-30.6)$ \\
\hline $\mathrm{DPI} \pm \mathrm{SD}(\mathrm{db})$, mean $\pm \mathrm{SD}$ (median, IQR) & $12.9 \pm 3.5(12.7,10.9-15.4)$ \\
\hline Time from peak to one half (s), mean \pm SD (median, IQR) & $64.1 \pm 26.3(58.1,47.6-73.7)$ \\
\hline $\mathrm{EI} \pm \mathrm{SD}(\mathrm{m} / \mathrm{s})$, mean $\pm \mathrm{SD}($ median, IQR) & $2.5 \pm 0.8(2.5,2.1-2.9)$ \\
\hline AUC of TIC (db·s), mean \pm SD (median, IQR) & $1170.6 \pm 1061.2(1034.8,825.3-1304.5)$ \\
\hline
\end{tabular}

GFR: glomerular filtration rate; BMI: Body mass index; MAP: mean arterial blood pressure; UPE: 24-h urinary protein excretion; Scr: Serum creatinine; RI: resistance index; AT: arrival time; TTP: time to peak; DPI: derived peak intensity; EI: elastic index; AUC of TIC, area under time-intensity curves

Table 2. Ultrasound parameters influencing composite kidney failure events by univariate and multivariate Cox regression in CKD patients who had CEUS to detect and predict renal function changes 


\begin{tabular}{|c|c|c|c|c|c|c|}
\hline \multirow[t]{2}{*}{ Parameters } & \multicolumn{3}{|c|}{ Univariate } & \multicolumn{3}{|c|}{ Multivariate } \\
\hline & HR & $95 \% \mathrm{CI}$ & $\mathrm{p}$ value & HR & $95 \% \mathrm{CI}$ & $\mathrm{p}$ value \\
\hline Age (years) & 1.05 & $1.02-1.08$ & $<0.001$ & & & 0.39 \\
\hline $\operatorname{BMI}\left(\mathrm{kg} / \mathrm{m}^{2}\right)$ & 1.01 & $0.91-1.11$ & 0.89 & & & 0.80 \\
\hline eGFR (ml/min per $\left.1.73 \mathrm{~m}^{2}\right)$ & 0.98 & 0.97-0.99 & 0.01 & & & 0.99 \\
\hline MAP (mmHg) & 1.02 & $0.99-1.05$ & 0.23 & & & 0.24 \\
\hline HbA1c (\%) & 1.32 & $1.13-1.53$ & $<0.001$ & 1.71 & $1.06-2.76$ & 0.03 \\
\hline UPE (mg/L) & 1.21 & $1.11-1.32$ & $<0.001$ & 1.24 & $1.12-1.37$ & $<0.001$ \\
\hline Length (mm) & 1.04 & $1.00-1.08$ & 0.09 & & & \\
\hline Width (mm) & 1.06 & $1.01-1.13$ & 0.03 & & & 0.77 \\
\hline Cortical thickness (mm) & 0.80 & $0.68-0.95$ & 0.01 & & & 0.70 \\
\hline RI (g/dL) & 30448.9 & $0.96-961893439.5$ & 0.05 & & & \\
\hline $\mathrm{AT}(\mathrm{s})$ & 1.05 & $0.98-1.12$ & 0.19 & & & \\
\hline TTP(s) & 1.07 & $1.02-1.12$ & 0.003 & & & 0.37 \\
\hline DPI (db) & 0.85 & $0.76-0.95$ & 0.003 & 0.86 & $0.74-0.99$ & 0.03 \\
\hline Time from peak to one half (s) & 0.99 & $0.97-1.01$ & 0.14 & & & \\
\hline $\mathrm{EI}(\mathrm{m} / \mathrm{s})$ & 1.59 & $1.07-2.35$ & 0.02 & & & 0.20 \\
\hline AUC of TIC $(\mathrm{db} \cdot \mathrm{s})$ & 1.00 & $1.00-1.00$ & 0.49 & & & \\
\hline
\end{tabular}

HR, hazard ratio, 95\% CI, 95\% confidence interval.

Table 3. AUC of DPI, UPE, HbA1c and parameter combination for renal prognosis prediction of CKD patients who had CEUS

\begin{tabular}{|c|c|c|c|c|c|c|c|}
\hline & ROC-AUC & $\begin{array}{c}\text { Sensitivity } \\
(\%)\end{array}$ & $\begin{array}{c}\text { Specificity } \\
\text { (\%) }\end{array}$ & PPV (\%) & NPV (\%) & $\mathrm{LR}+$ & LR- \\
\hline $\begin{array}{l}\text { Parameter } \\
\text { combination }\end{array}$ & $\begin{array}{l}0.80(0.73- \\
0.86)\end{array}$ & $\begin{array}{c}77.8 \\
(57.7-91.4)\end{array}$ & $\begin{array}{c}75.4 \\
(66.9-82.6)\end{array}$ & $\begin{array}{c}40.3 \\
(27.0-54.9)\end{array}$ & $\begin{array}{c}94.1 \\
(87.5-97.8)\end{array}$ & $\begin{array}{c}3.2 \\
(2.2-4.6)\end{array}$ & $\begin{array}{c}0.3 \\
(0.1- \\
0.6)\end{array}$ \\
\hline DPI (db) & $\begin{array}{c}0.65 \\
(0.57-0.72)\end{array}$ & $\begin{array}{c}65.5(45.7- \\
82.1)\end{array}$ & $\begin{array}{c}61.5(52.7- \\
69.7)\end{array}$ & $\begin{array}{l}27.2(17.3- \\
39.0)\end{array}$ & $\begin{array}{c}89.0(80.9- \\
94.6)\end{array}$ & $\begin{array}{c}1.7(1.2- \\
2.4)\end{array}$ & $\begin{array}{l}0.6 \\
(0.3- \\
0.9)\end{array}$ \\
\hline $\mathrm{UPE}(\mathrm{g} / \mathrm{L})$ & $\begin{array}{c}0.69 \\
(0.61-0.76)\end{array}$ & $\begin{array}{c}77.8 \\
(57.7-91.4)\end{array}$ & $\begin{array}{c}65.4 \\
(56.8-73.4)\end{array}$ & $\begin{array}{c}30.9 \\
(20.2-43.2)\end{array}$ & $\begin{array}{c}93.7 \\
(86.8-97.6)\end{array}$ & $\begin{array}{c}2.3 \\
(1.7-3.1)\end{array}$ & $\begin{array}{l}0.3 \\
(0.2- \\
0.7)\end{array}$ \\
\hline HbA1c (\%) & $\begin{array}{c}0.63 \\
(0.51-0.75)\end{array}$ & $\begin{array}{c}48.3 \\
(29.4-67.5)\end{array}$ & $\begin{array}{c}77.9 \\
(69.8-84.6)\end{array}$ & $\begin{array}{c}52.6 \\
(28.9-75.5)\end{array}$ & $\begin{array}{c}86.5 \\
(79.8-91.7)\end{array}$ & $\begin{array}{c}2.2 \\
(1.3-3.6)\end{array}$ & $\begin{array}{l}0.7 \\
(0.5- \\
1.0)\end{array}$ \\
\hline
\end{tabular}


Statistical quantifications were demonstrated with 95\% CI, when applicable.

NPV, negative predictive value; PPV, positive predictive value; LR+, positive diagnostic likelihood ratio; LR-, negative diagnostic likelihood ratio.

Table 4. Correlation between DPI and clinical factors of CKD patients who had CEUS to detect and predict renal function changes

\begin{tabular}{lccc}
\hline \multicolumn{1}{c}{ Characteristics } & DPI $>11.98 \mathrm{db}(\mathrm{n}=96)$ & $\mathrm{DPI} \leq 11.98 \mathrm{db}(\mathrm{n}=71)$ & P value \\
\hline Age (years) & $41.0(29.5,56.75)$ & $46.0(30.0,56.0)$ & 0.54 \\
Men, n(\%) & $46(47.9)$ & $41(57.7)$ & 0.21 \\
BMI (kg/m²) & $24.2(21.9,25.7)$ & $25.0(22.7,27.3)$ & 0.02 \\
Baseline MAP (mmHg) & $93.3(87.3,105.3)$ & $96.0(90.1,103.3)$ & 0.86 \\
HgbA1c (\%) & $5.3(5.1,5.5)$ & $5.4(5.1,6.0)$ & 0.08 \\
UPE (g/L) & $1.8(0.8,4.0)$ & $1.8(0.8,4.0)$ & 0.66 \\
SCr ( $\mathrm{Mmol} / \mathrm{l})$ & $78.3(64.5,95.3)$ & $91.5(65.6,125.2)$ & 0.01 \\
eGFR (ml/min per 1.73m $\left.{ }^{2}\right)$ & $92.4(69.5,106.8)$ & $78.3(51.9,107.6)$ & 0.02 \\
$\alpha-S M A(\%)$ & $1.24(0.78,2.14)$ & $1.95(1.35,3.09)$ & $<0.001$ \\
\hline
\end{tabular}

\section{Figures}




\section{Patients with CKD underwent examination of CEUS}

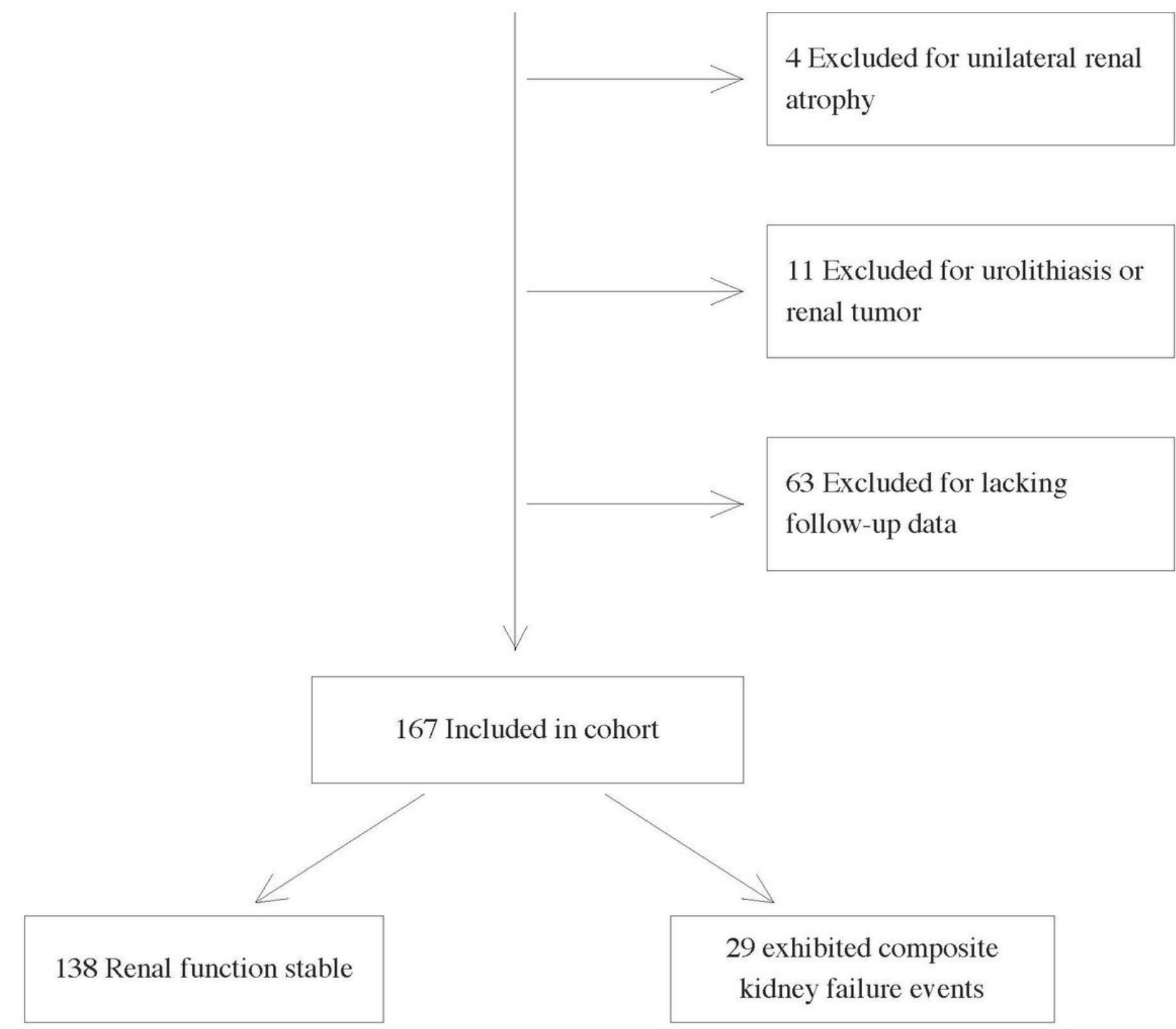

\section{Figure 1}

Flowchart of the cohort of chronic kidney disease (CKD) patients who had contrast-enhanced ultrasonography (CEUS) to detect and predict renal function changes (Composite kidney failure event included eGFR halving compared to baseline values or entering ESRD. In total, 167 out of 245 patients were enrolled in this study. CKD, chronic kidney disease; CEUS, contrast-enhanced ultrasound.) 


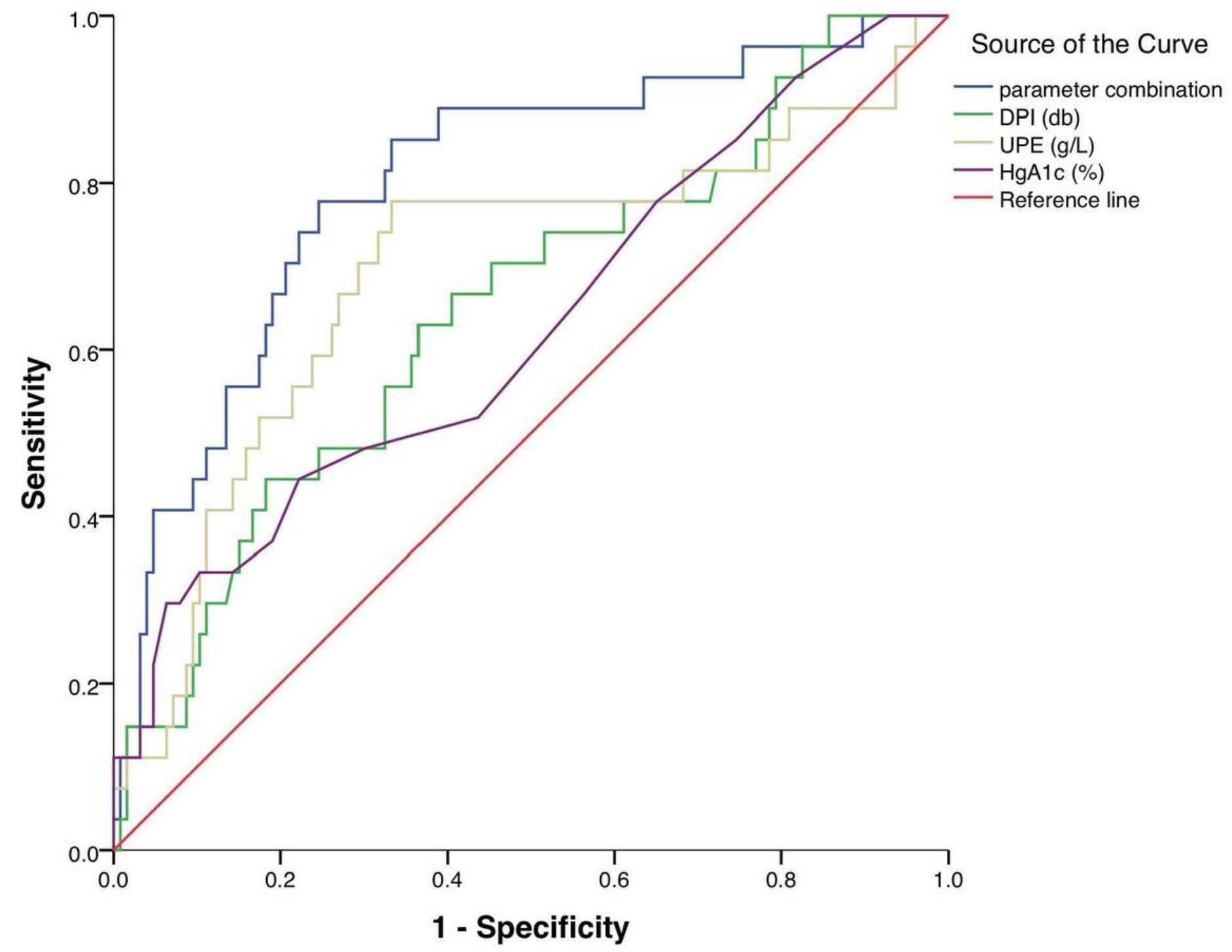

Figure 2

Receiver operating characteristic (ROC) for renal prognosis prediction. Comparison of ROC curve between $\mathrm{DPI}, \mathrm{UPE}, \mathrm{HbA} 1 \mathrm{c}$ and parameter combination. Parameter combination: combining DPI, UPE and HbA1c. The cut off value of DPI was $11.98 \mathrm{db}$. 


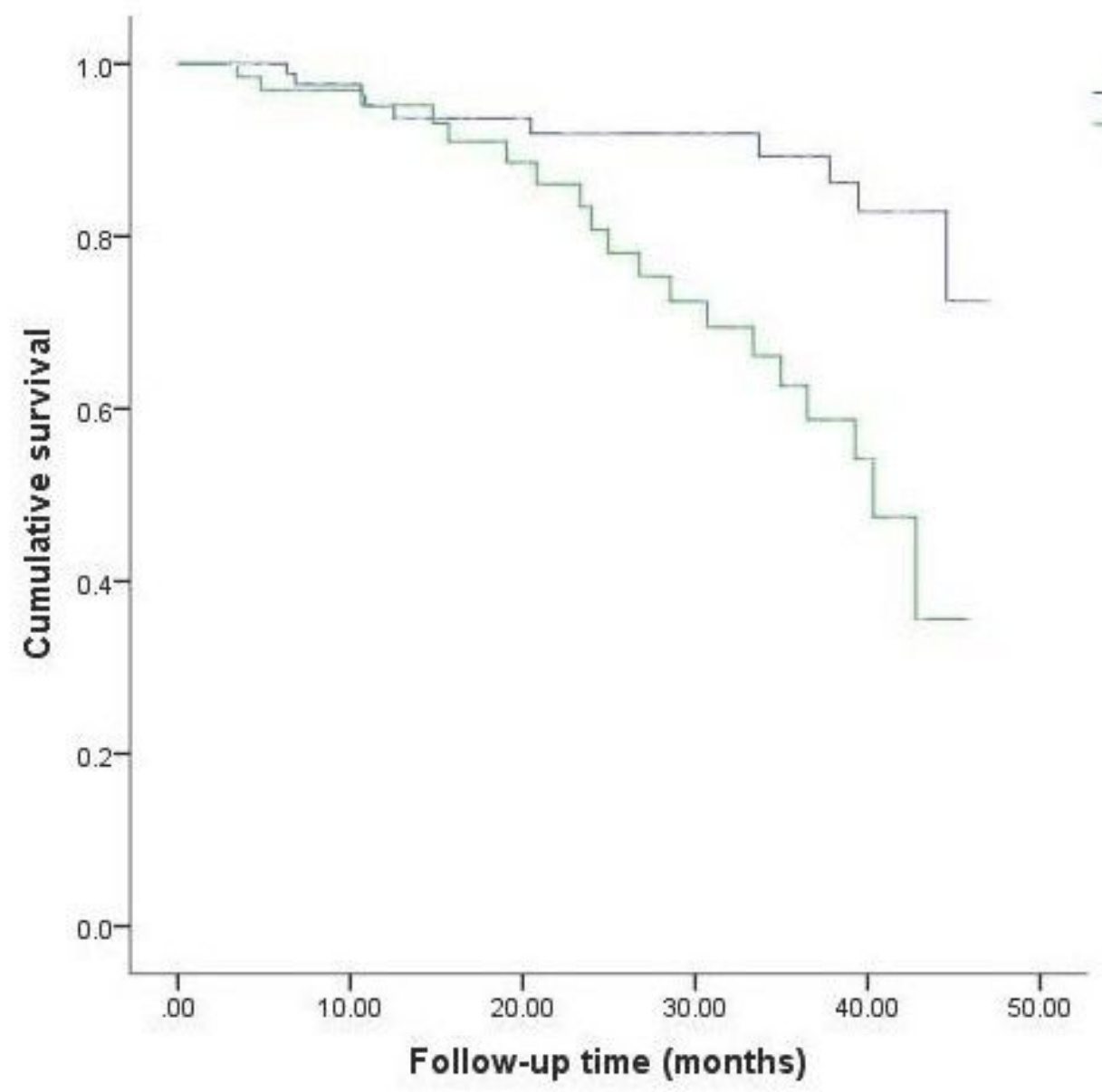

\section{Figure 3}

Kaplan-Meier plots showing renal survival stratified by the cut off value of DPI of CKD patients who had CEUS to detect and predict renal function changes

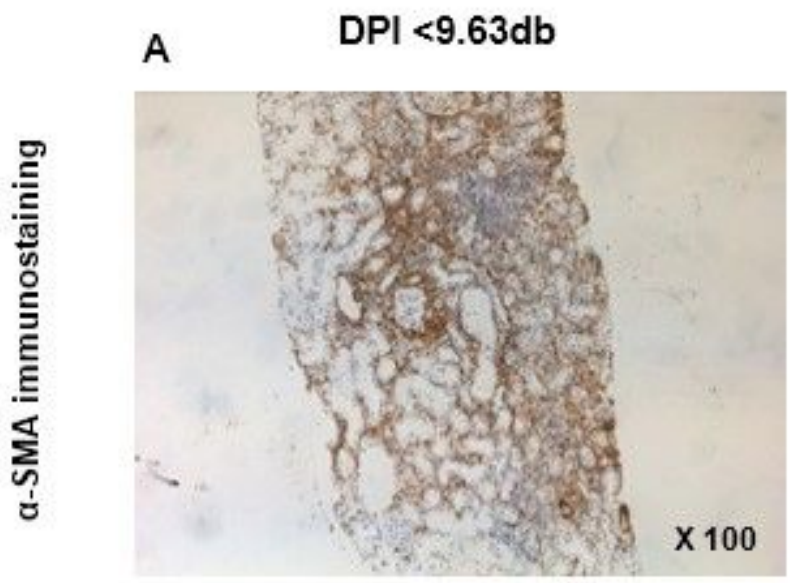

B $\quad$ DPI $\geq 9.63 \mathrm{db}$

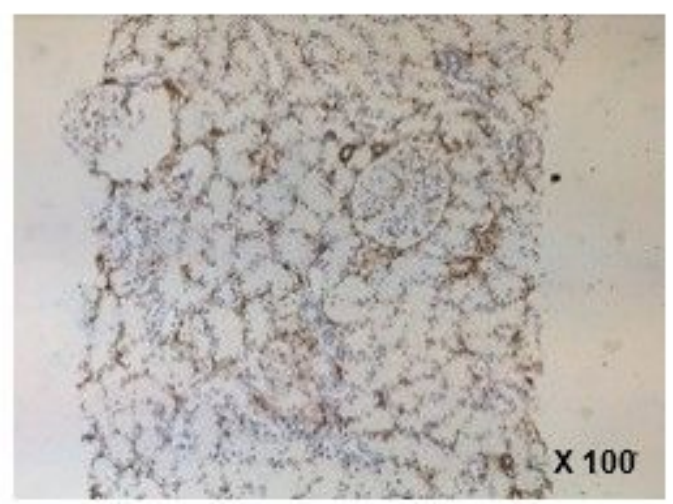

Figure 4

Renal interstitial fibrosis compared between groups with different DPI levels. (A) and (B) a-SMA immunostaining $(X 100)$. *, $p<0.001$. DPI, derived peak intensity. Compared to the group with a higher DPI, 
the group with a DPI less than $11.98 \mathrm{db}$ exhibited increased renal fibrosis.

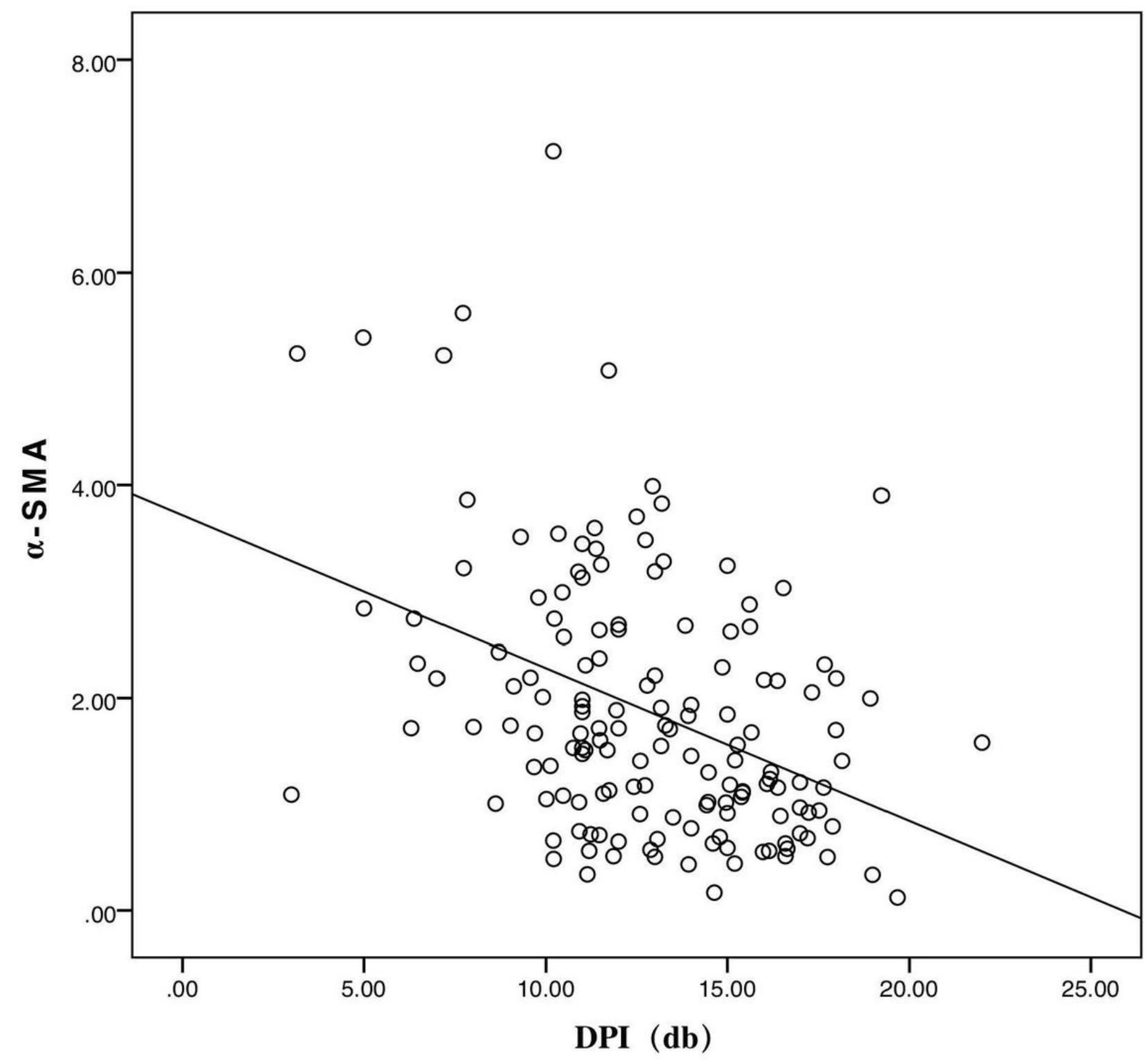

Figure 5

Spearman correlation analysis for DPI level and renal fibrosis. DPI levels are negatively correlated with the degree of renal fibrosis according to a-SMA expression. $(r=-0.365, p<0.001)$ 


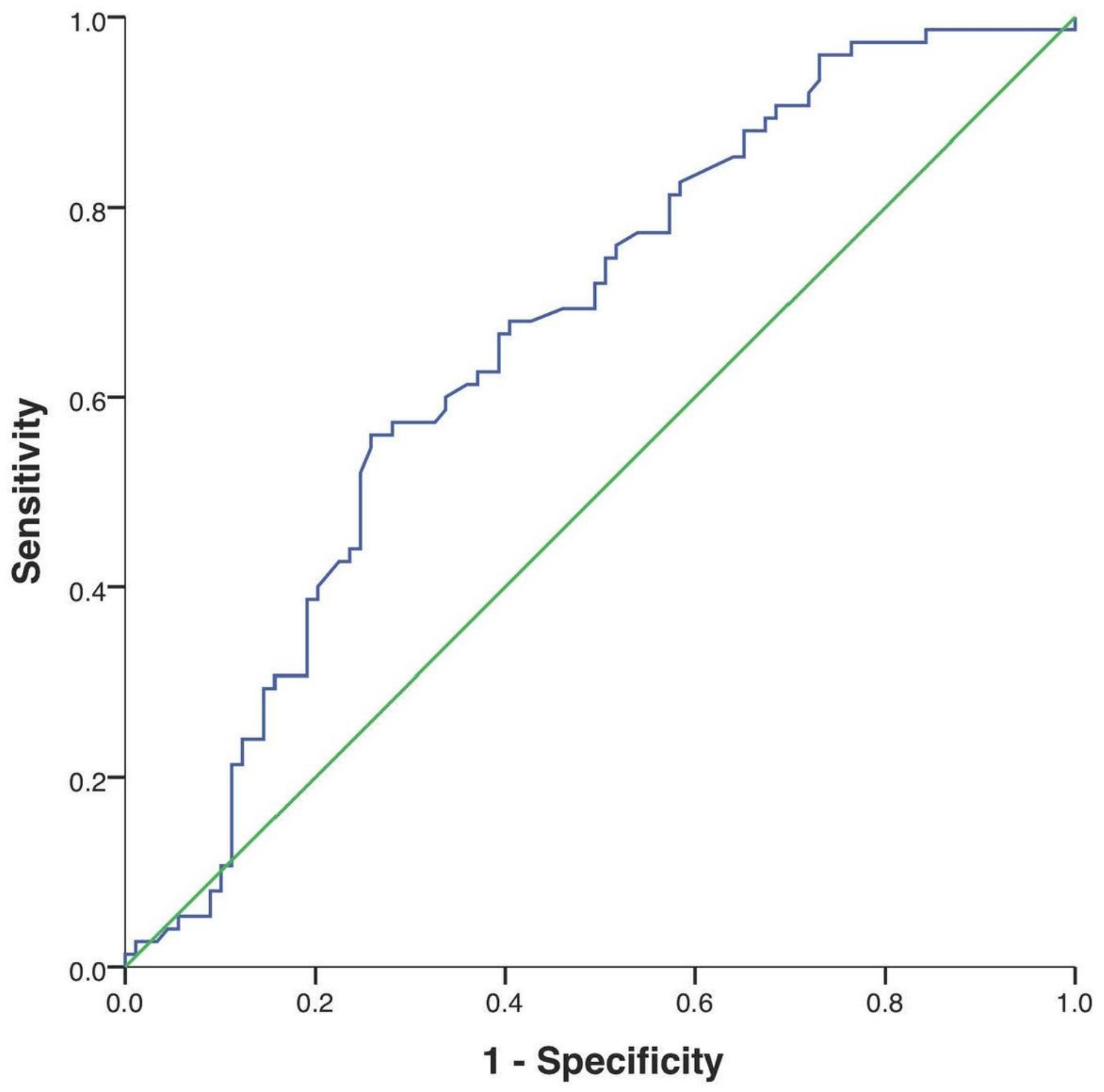

Figure 6

The ROC for renal fibrosis prediction. Relationship between DPI level and renal fibrosis. Corresponding AUC is 0.67 (95\% confidence interval $(\mathrm{Cl}), 0.58-0.75, \mathrm{P}<0.001)$ with a sensitivity of $67 \%$ and a specificity of $61 \%$. 